\title{
Sparse Parameter Modeling and Estimation for Trajectory Process Based on Semi- parameter Regression Analysis
}

\author{
Chunlin Huang \\ PLA Forces 92941 \\ Huludao, China, 125001
}

\begin{abstract}
The measuring model based on Bi-satellite as well as optical and radar measure was presented; and then the sparse parameter estimation model and the corresponding estimation algorithm and calculation step for exterior ballistic trajectory based on semi-parameter regression model was established. Moreover this paper analyzed and explained that this algorithm can eliminate the effect on the estimation precision of trajectory parameters which were brought by the nonlinear ingredients more effectively than the least square method both from theory analysis and simulation experiments.
\end{abstract}

Keywords-Exterior ballistic trajectory, Estimation and Modeling, Semi-parameter regression, Precision analysis

\section{INTRODUCTION}

Along with the increase of the number of satellites and the improvement of the space-based monitoring and control system, the navigation system plays an increasingly important role. GPS [1] is of great importance in current tests of weapons and equipment. However, the Bi-satellite system is of increasing importance in tests of the weapons and equipment along with its improvement. At present, the optical tracking system, the pulse radar, GPS and the Bisatellite positioning system serve as basic measuring means for obtaining the tracking data and flight state of an exterior ballistic trajectory [2]. Estimation of the exterior ballistic trajectory means utilizing a large number of measurement data to obtain trajectory estimation results via an appropriate non-linear parameter estimation algorithm and includes two modeling processes: an observation model and an estimation model. The trajectory estimation, therefore, can be boiled down to multi-model nonlinear parameter estimation, which can be solved in such ways as an EMBET method, a least squares method and a sparse parameter modeling method [3] which generally need model linearization and attribute truncation errors to noise, however, nonlinear factors always exist in a real model, such as truncation errors discarded after linearization of observation equations, various types of system errors and random interference factors existing in observation data, outliers which are not eliminated in preprocessing of initial data, etc. There are also a large number of means to achieve better results, such as the robust theory [4], the precision factor conversion method [5] and the like, but they have a variety of defects and can affect the feasibility and credibility.

The semi-parameter regression model [6,7] can effectively overcome the defects of nonlinear treatment mentioned above, is closer to reality, and can make full use of information provided in data. Based on the trajectory estimation principle, the paper presents semi-parameter regression modeling for two trajectory estimation models. For the observation model, linear ingredients correspond to trajectory estimation ingredients, nonlinear ingredients come down to observation residuals, nonlinear factors can be obtained via removing random noise, and the nonlinear estimation of observation residuals based on a polynomial modulating function can be established based on analysis of an atmospheric refraction correction model; and for the estimation model, a method is proposed for iteration of linear estimation and nonlinear estimation. But there are some problems related to the semi-parametric model:

(1) The early elimination of nonlinear errors may cause deletion of critical data and affect the accuracy of iterative calculation of follow-up parameters; and

(2) Difficulties in parameter estimation may be caused as a result of too many parameters for calculation.

Though few parameters are used for estimation of the whole trajectory in the sparse parameter model, its parameter estimation is on the basis that all models with errors have been described and then approximation is carried out. Errors may occur to approximation in the real error model calculation as a result of uncertain errors and truncation errors. In combination of the advantages and disadvantages of the two methods, the paper establishes a sparse parameter model based on semi-parametric regression and a corresponding parameter estimation method according to the status of the shooting range, and effectively eliminates the influence of nonlinear factors on ballistic trajectory estimation. Finally, the simulation data processing and accuracy analysis show that the algorithm really works.

\section{PARAMETER ESTIMATION MODEL AND ALGORITHM}

\section{A. Sparse Ballistic Trajectory Parameter Model}

During target tracking, some factors of the ballistic trajectory are known, such as inter-stage shutdown points and ignition points. Due to their poor differentiability of ballistic trajectory parameters $(x(t), y(t), z(t), \dot{x}(t), \dot{y}(t), \dot{z}(t))$ in a launching coordinate system, these points are referred to as feature points. Practice shows that when the feature points are used as spline knot points, more than one third of parameters can be saved.

Taking sampling data at $t_{1}, t_{2}, \ldots, t_{m}$ into consideration, if $\mathrm{r}$ features points are within $\left[t_{1}, t_{m}\right]\left(t_{1} \leq T_{1}<T_{\ldots} \ldots T_{r} \leq t_{m}\right)$, and three- 
spline are used to describe the ballistic trajectory, the spline knot points describing the ballistic trajectory meet the condition: $T_{1}<T_{2}<T_{3}<T_{4}=t_{1}<\ldots<T_{M+1}=t_{m}<T_{M+2}<T_{M A 3}<T_{M 44}$, where M means the number of knot points in the splice function. When $\psi_{j}(t)$ is set as the spline basis function, the trajectory function is expressed as:

$$
\left\{\begin{array}{l}
x(t)=\sum_{p=1}^{M} d_{p} \psi_{p}(t), \quad \dot{x}(t)=\sum_{p=1}^{M} d_{p} \dot{\psi}_{p}(t) \\
y(t)=\sum_{p=1}^{M} d_{p+M} \psi_{p}(t), \quad \dot{y}(t)=\sum_{p=1}^{M} d_{p+M} \dot{\psi}_{p}(t), \quad t_{1} \leq t \leq t_{m} \\
z(t)=\sum_{p=1}^{M} d_{p+2 M} \psi_{p}(t), \dot{z}(t)=\sum_{p=1}^{M} d_{p+2 M} \dot{\psi}_{p}(t)
\end{array}\right.
$$

Where, $\boldsymbol{\beta}=\left(d_{1}, d_{2}, \cdots, d_{3 M}\right)$ is a coefficient of the spline function.

\section{B. Nonlinear Fitting of Observation Equation and Observation Residual}

The following measurement equation can be obtained according to operating principles of the Bi-satellite and optical radar equipment:

$$
\left\{\begin{aligned}
Y_{1}(t) & =2 R_{1}(t)+2 r_{1}(t)+e_{1}(t) \\
& =G_{1}(\boldsymbol{X}(t))+e_{1}(t) \\
Y_{2}(t) & =R_{1}(t)+R_{2}(t)+r_{1}(t)+r_{2}(t)+e_{2}(t) \\
& =G_{2}(\boldsymbol{X}(t))+e_{2}(t) \\
A_{i}(t) & =\operatorname{arctg} \frac{z(t)}{x(t)}+r_{A i}(t)+e_{A i}(t) \\
& =A_{i}(\boldsymbol{X}(t))+e_{A i}(t) \\
E_{i}(t) & =\operatorname{arctg} \frac{y(t)}{\sqrt{x^{2}(t)+z^{2}(t)}}+r_{E i}(t)+e_{E i}(t) \\
& =E_{i}(\boldsymbol{X}(t))+e_{E i}(t) \\
R_{i}(t) & =r_{i}(t)+r_{R i}(t)+e_{R i}(t)=R_{i}(\boldsymbol{X}(t))+e_{R i}(t)
\end{aligned}\right.
$$

Where, $Y_{1}(t), Y_{2}(t)$ indicate the Bi-satellite distance and the observed quantity; $A_{i}(t), E_{i}(t), R_{i}(t)$ respectively indicate the observed quantity of the azimuth, altitude and distance of a user relative to a monitoring station $\mathrm{i}$ at $\mathrm{t} ; r_{1}(t), r_{2}(t)$ indicate the distances from the user to two static satellites; $R_{1}(t), R_{2}(t)$ indicate the distances from a ground central station to the two satellites, and $r_{i}(t)$ indicates the distance from the user to the monitoring state $\mathrm{I}$ and is a function of the distances from the user to the satellites; $\boldsymbol{X}(t)=(x(t), y(t), z(t))$ is a position factor of the user relative to the satellites and is a factor to be determined, and $r_{A i}, r_{E i}$ and $r_{R i}$ respectively indicate nonlinear uncertain factors of observation of the azimuth, altitude and distance; and $e_{1}, e_{2}, e_{A i}, e_{E i}, e_{R i}$ indicate total observation errors of the

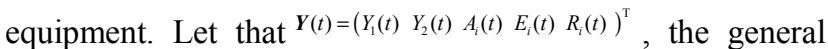
treatment of the observation equation means expanding the Taylor at a nominal position $\boldsymbol{X}^{*}$ of an initial value of a state vector, then:

$$
\boldsymbol{Y}(t)=\boldsymbol{F}\left(\boldsymbol{X}^{*}(t), t\right)+\left.\frac{\partial \boldsymbol{F}}{\partial \boldsymbol{X}}\right|_{X=X^{\prime}(t)}\left(\boldsymbol{X}(t)-\boldsymbol{X}^{*}(t)\right)+\varepsilon(t), \text {, Let }\left\{\begin{array}{l}
\Delta \boldsymbol{y}=\boldsymbol{Y}(t)-\boldsymbol{F}\left(\boldsymbol{X}^{*}(t), t\right) \\
\Delta \boldsymbol{x}=\boldsymbol{X}(t)-\boldsymbol{X}^{*}(t) \\
\boldsymbol{H}=\left.\frac{\partial \boldsymbol{F}}{\partial \boldsymbol{X}}\right|_{X=X^{*}(t)}
\end{array}\right.
$$

Then the observation equation constitutes a linear system $\Delta \boldsymbol{y}=\boldsymbol{H} \Delta \boldsymbol{x}+\boldsymbol{\varepsilon}$, where $\boldsymbol{\varepsilon}$ indicates a random measurement error vector.

In practice, however, the linearization of the observation equation depends on the selection of the initial trajectory and certainly has truncation errors; and the observation data are also subject to the interference of various types of system errors and random uncertainties and abnormal values which are not removed in initial data pre-processing. In the traditional linearization method, these nonlinear factors are all attributed to the random error $\boldsymbol{\varepsilon}$, which is obviously inconsistent with the actual situation and can influence the accuracy of trajectory estimation. Hence, the deep nonlinear estimation is needed for the observed value and the calculated value, so as to separate systematic errors and random errors and improve the accuracy of the observed data.

Nonlinear estimation for observation residuals can be carried out based on analysis of ranging residuals and measurement angles of ground points and in combination of the atmospheric radio wave refraction correction model, and the following residual fitting function [3] of the corresponding observed quantity in the observation equation (2) is used:

$$
\begin{aligned}
& \left\langle\Delta Y_{1}(t)=a_{01} \csc \left(a_{11}+a_{21} t+a_{31} \cos \left(\omega_{11} t\right)+a_{41} \sin \left(\omega_{21} t\right)\right)\right. \\
& \Delta Y_{2}(t)=a_{02} \csc \left(a_{12}+a_{22} t+a_{32} \cos \left(\omega_{12} t\right)+a_{42} \sin \left(\omega_{22} t\right)\right) \\
& \Delta R_{i}(t)=b_{i 1}+b_{i 2} t+\left(b_{i 3}+b_{i 4} t\right) R_{i}(t) \\
& +b_{i 5} \dot{R}_{i}(t)+b_{i 6} \csc E_{i}(t) \\
& \Delta A_{i}(t)=c_{i 1} \sin A_{i}(t) \tan E_{i}(t)+c_{i 2} \tan E_{i}(t) \\
& +c_{i 3} \sec E_{i}(t)+\left(c_{i 4} / f_{i}\right) \cos E_{i}(t) \\
& \Delta E_{i}(t)=c_{i 1} \cos A_{i}(t)+c_{i 5} \cos E_{i}(t)+c_{i 6} / f_{i} \\
& \boldsymbol{\Delta r}(t)=\left[\begin{array}{c}
\Delta Y_{1}(t) \\
\Delta Y_{2}(t) \\
\Delta R_{i}(t) \\
\Delta A_{i}(t) \\
\Delta E_{i}(t)
\end{array}\right]
\end{aligned}
$$

Where:

$\boldsymbol{s}=\left(a_{01}, a_{11}, a_{21}, a_{31}, a_{41}, a_{02}, a_{12}, a_{22}, a_{22}, a_{42}, b_{11}, b_{12}, b_{13}, b_{i 4}, b_{i 5}, b_{i 6}, c_{11}, c_{i 2}, c_{13}, c_{i 4}, c_{i 5}, c_{i 6}\right) \quad i=1,2, \cdots, N$ in dicates parameters of a system error model to be estimated; $\mathrm{N}$ indicates the number of monitoring stations; $t$ indicates the observation time; ${ }^{f_{i}}$ indicates the known focal length of the optical system; and $\omega_{11}, \omega_{21}, \omega_{12}, \omega_{22}$ indicate the frequency of a periodic function to be estimated.

The following basis function fitting is carried out for an observation residual of a specific ballistic trajectory. It shall be checked prior to fitting whether the residual is white noise. If the residual is white noise, there is no need to extract nonlinear factors in the residual via basis function fitting; and if the residual is not white noise, nonlinear factors shall be extracted via fitting. Figure 1 shows the comparison of the

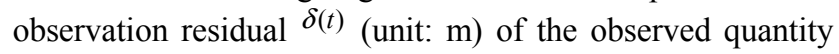


$Y_{1}(t)$ of the Bi-satellite distance and its fitting function ${ }^{\Delta Y_{1}(t)}$. Figure 2 shows the difference Delta (unit: $m$ ) of the observation residual and a function fitting residual, which is closer to a white noise error.

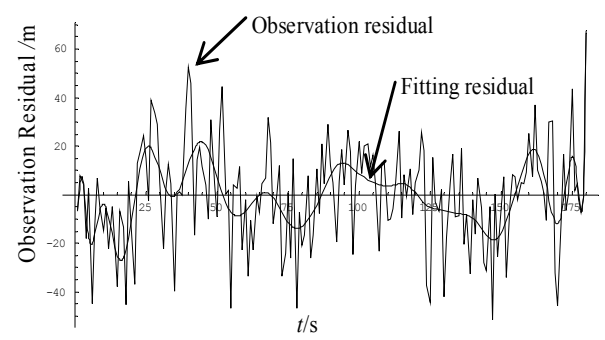

Figure 1. Observation Residual and Fitting Residual

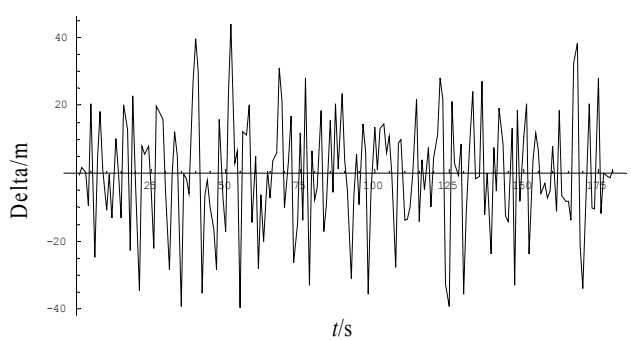

Figure 2. Differences of Two Residuals

It can be seen that large system errors really exist in the observation residual, and system error factors in the residuals can be separated effectively via nonlinear estimation with the trigonometric function and the polynomial fitting observation residual, so that the rest tend to be white noise. Therefore, it can be concluded that the fitting of the observation residual with the basis function is equivalent to estimation of the nonlinear function, the residual is considered colored noise, and the white noise is left when the nonlinear factors are removed.

\section{Ballistic Trajectory Estimation Algorithm}

Based on the nonlinear fitting of residual sequences, the linear system related to the parameter $\beta$ to be estimated in the observation model can be described as follows:

$$
\boldsymbol{Y}(t)=\boldsymbol{F}(\boldsymbol{\beta}, t)+\Delta \boldsymbol{r}(t)+\boldsymbol{\varepsilon}
$$

Where ${ }^{\Delta r(t)}$ indicates a fitting function of the observation residual, and $\boldsymbol{\varepsilon}$ indicates Gaussian white noise. Thus a ballistic trajectory estimation model based on semiparameter regression is established.

Considering the spline coefficients, system error parameters and semi-parameters in the estimation of the model and according to the theory of nonlinear semiparameter regression analysis, the spline coefficients and the system error parameters can be estimated via the solution of a extreme value problem

$$
\arg \min _{\beta \in R^{R}} \sum_{j=1}^{m} \rho_{j} \sigma_{j}^{-2}\left\|\boldsymbol{Y}_{j}-\boldsymbol{F}_{j}(\boldsymbol{\beta})-\boldsymbol{S}_{j}\right\|^{2}
$$

Where ${ }^{\rho_{j}}$ indicates the observed weight at $\mathrm{j},{ }^{\sigma_{j}}$ indicates the corresponding observed variance, and ${ }^{S_{j}}$ indicates the observed system error.

Taking nonlinear factors of the semi-parameter model into account $s$ can be fitted via the following polynomial modulating function:

$$
S(t)=\sum_{k=0}^{m-1}\left(\sum_{l=0}^{N_{k}-1} a_{i}^{(k)} t^{l}\right) \cdot \exp \left\{j 2 \pi v_{k} t\right\} \stackrel{\text { def }}{=} \sum_{k=0}^{m-1} b_{k}(t) \cdot \exp \left\{j 2 \pi v_{k} t\right\}
$$

Where, ${ }^{v_{k}}$ indicates the frequency of the nonlinear factors, $b_{k}(t)$ indicates the amplitude envelope of each component signal, and $a_{i}^{(k)}$ indicates the polynomial coefficient corresponding to the envelope function.

The algorithm steps for determination of the parameters to be estimated are as follows:

Step 1: Based on a group of given weights $\boldsymbol{\rho}^{(0)}$ and a given initial value of iteration $\boldsymbol{\beta}^{(0)}$, obtaining $\boldsymbol{F}_{j}\left(\boldsymbol{\beta}^{(0)}\right)$ via a functional expression of a measuring element related to the parameters to be estimated.

Step 2: Calculating the gradient matrix $V_{j}\left(\beta^{(0)}\right)$ [8] of $\boldsymbol{F}_{j}\left(\boldsymbol{\beta}^{(0)}\right)$, carrying out $Q R$ decomposition of the gradient matrix, so that $Q^{T} Q=Q \Phi$ can serve as a unit matrix, $R=\left(\begin{array}{ll}R_{1} & 0\end{array}\right)^{T}$ is an upper triangular matrix, and the matrix $\boldsymbol{L}_{j}\left(\boldsymbol{\beta}^{(0)}\right)=R_{1}^{-1}$ can be obtained.

Step 3: Obtaining the primary improvement of the parameters via the following expression:

$$
\left\{\begin{array}{l}
\boldsymbol{D}^{(1)}=\left[\sum_{j=1}^{m} \boldsymbol{V}_{j}^{\mathrm{T}}\left(\boldsymbol{\beta}^{(0)}\right) \boldsymbol{V}_{j}\left(\boldsymbol{\beta}^{(0)}\right)\right]^{-1} \\
\quad \sum_{j=1}^{m} \sqrt{\boldsymbol{\rho}_{j}^{(0)}} \sigma_{j}^{-1} \boldsymbol{V}_{j}^{\mathrm{T}}\left(\boldsymbol{\beta}^{(0)}\right)\left[\boldsymbol{Y}_{j}-\boldsymbol{F}_{j}\left(\boldsymbol{\beta}^{(0)}\right)\right] \\
J\left(\boldsymbol{\beta}^{(0)}+\lambda_{1} \boldsymbol{D}^{(1)}\right)=\min _{0<\lambda<1} J\left(\boldsymbol{\beta}^{(0)}+\lambda \boldsymbol{D}^{(1)}\right) \\
\boldsymbol{\beta}^{(1)}=\boldsymbol{\beta}^{(0)}+\lambda_{1} \boldsymbol{D}^{(1)}
\end{array}\right.
$$

Where $\boldsymbol{D}^{(1)}$ indicates the improved quantity of the parameters in primary iteration; ${ }^{V_{j}}\left(\beta^{(0)}\right)$ indicates the gradient matrix of $\boldsymbol{F}_{j}\left(\boldsymbol{\beta}^{(0)}\right)$; and $\lambda$ indicates the step length of parameter improvement, ${ }^{\lambda}$ indicates the optimal step length within $\lambda \in(0,1), J$ indicates the objective optimization function. For the specific expression, see Reference [8].

Step 4: When the given convergence threshold $\tau>0$ and $J\left(\boldsymbol{\beta}^{(0)}\right)-\tau<J\left(\boldsymbol{\beta}^{(1)}\right)<J\left(\boldsymbol{\beta}^{(0)}\right)$, it is indicated that the iteration is completed; let $\tilde{\boldsymbol{\beta}}=\boldsymbol{\beta}^{(1)}$, carry out Step5; otherwise, let $\boldsymbol{\beta}^{(0)}=\boldsymbol{\beta}^{(1)}$, return to Step1;

Step 5: Calculating the second derivative threedimensional matrix $\boldsymbol{W}_{j}(\tilde{\boldsymbol{\beta}})$ of $\boldsymbol{F}_{j}(\tilde{\boldsymbol{\beta}})$, the three-dimensional matrix with an inherent curvature and the three-dimensional matrix of parameter effect, and obtaining $\boldsymbol{V}_{I}^{j}, \boldsymbol{V}_{P}^{j}$, and for the specific form, see Reference [8]; 
Step 6: Calculating the mean square error matrix $\operatorname{MSEM}(\tilde{\boldsymbol{\beta}})$ of the parameter estimate $\tilde{\boldsymbol{\beta}}$ and the minimum $\tilde{\boldsymbol{\rho}}$ via similar iteration;

Step 7: Let $\boldsymbol{\rho}^{(0)}=\tilde{\boldsymbol{\rho}}, \quad \boldsymbol{\beta}^{(0)}=\tilde{\boldsymbol{\beta}}$, detecting whether the residual is white noise, and if the residual is white noise, carrying out Step9;

Step 8: Determining the vector of nonlinear factors in an aliasing frequency estimation method $\boldsymbol{S}=\left(\boldsymbol{S}_{1}\left(t_{j}\right), \boldsymbol{S}_{2}\left(t_{j}\right), \ldots \boldsymbol{S}_{N}\left(t_{j}\right)\right)^{\mathrm{T}} j=1, \ldots, m$, where $\boldsymbol{S}_{j}$ meets: $\boldsymbol{Y}_{j}-\boldsymbol{F}_{j}\left(\boldsymbol{\beta}^{(0)}\right)=\boldsymbol{S}_{j}+\boldsymbol{\varepsilon} \quad j=1,2, \ldots, m, \quad \varepsilon \quad$ indicates a white noise sequence, substituting $\boldsymbol{Y}-\boldsymbol{F}\left(\boldsymbol{\beta}^{(0)}\right)$ with $\boldsymbol{Y}-\boldsymbol{F}\left(\boldsymbol{\beta}^{(0)}\right)-\boldsymbol{\Delta} \boldsymbol{r}(t)$, and go to Step1.

Step 9: detecting whether parameters $\boldsymbol{\rho}, \boldsymbol{\beta}$ are subject to iterative convergence, convergence can be accepted, and the iterative results are recorded as $\hat{\boldsymbol{\rho}}, \hat{\boldsymbol{\beta}}$, which are the required solutions; otherwise, go to Step 1.

\section{ANALYSIS OF SimUlation RESUlts}

The simulation calculation and the analysis of results for estimation of the exterior ballistic trajectory are carried out based on the analysis of sparse parameter estimation modeling for the exterior ballistic trajectory based on the semi-parameter regression, including the fitting of observation residuals and the design of the ballistic trajectory estimation algorithm of the semi-parameter regression model.

Simulation conditions: for state model, STK is applied to simulate the theoretical ballistic trajectory; and for the observation model, the observation is carried out with three stations, three optical measurement devices and the $\mathrm{Bi}$ satellite system.

Simulation results and explanations: different error models are added into the observation model to produce observation data, and then the simulation observation data are adopted for ballistic trajectory estimation. For the three optical measurement devices, the 0.001-degree random error and the periodic colored noise with a 0.001 -degree amplitude are added into the simulation observation data; and the 2meter random error and periodic colored noise with a 1meter amplitude are added into the distance and the simulation observation data. Table I and Figure 3 shows the comparison of the multi-model multi-parameter estimation method (Method 1) [8] and the sparse parameter method based on semi-parameters (one of them adopts wavelet-based fitting (Method 2), and the other one adopts spline fitting (Method 3)).

TABLE I. ESTIMATION PRECISION COMPARISON AMONG THREE MODELS

\begin{tabular}{|c|c|c|c|c|}
\hline \multicolumn{2}{|c|}{ Estimation results } & $\mathbf{x} / \mathbf{m}$ & $\mathbf{y} / \mathbf{m}$ & $\mathbf{z} / \mathbf{m}$ \\
\hline \multirow{2}{*}{ Model 1 } & Mean & 2.15 & -6.34 & -4.48 \\
\cline { 2 - 5 } & Variance & 0.291 & 0.412 & 0.193 \\
\hline \multirow{2}{*}{ Model 2 } & Mean & -0.121 & 0.024 & -0.096 \\
\cline { 2 - 5 } & Variance & 0.206 & 0.142 & 0.131 \\
\hline \multirow{2}{*}{ Model 3 } & Mean & 0.015 & -0.147 & 0.079 \\
\hline
\end{tabular}

\begin{tabular}{|l|l|l|l|l|}
\hline & Variance & 0.249 & 0.371 & 0.186 \\
\hline
\end{tabular}

The follow conclusions can be drawn from the simulation results:

(1) If the solution of the observed quantity is obtained based on the semi-parameter regression calculation model, the precision of the solution results is higher than that of the multi-model multi-parameter fusion estimation method;

(2) If different fitting functions are adopted in the solution of the semi-parameter regression model, different results can be obtained. Since wavelets generally are advantageous for data fitting, better calculation results can be obtained generally when appropriate wavelets are used.
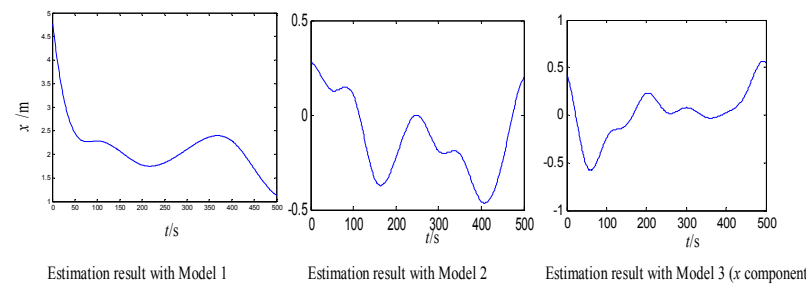

Estimation result with Model 3 ( $x$ component)

Figure 3. Estimation precision comparison in $\mathrm{x}$ component among three models

\section{SUMMARIES}

It is entirely feasible to establish a combined measuring model based on the Bi-satellite/ground base for measurement and estimation of the ballistic trajectory. The method has been used in the shooting range at present, which utilizes the existing resources more reasonably and fully, effectively achieves high utilization efficiency of the Bi-satellite and provides support for space-based monitoring and control.

Taking the complexity and uncertainty of measurement data of the equipment into account, the system error model can be established according to features of various types of equipment so as to effectively separate the influence of error factors. As for nonlinear factors in ballistic trajectory estimation, the sparse parameter fusion algorithm based on semi-parameter regression can effectively separate nonlinear factors in the observation residuals and improve the precision of the observation data. Therefore, the precision of ballistic trajectory estimation is greatly improved.

\section{REFERENCES}

[1] Sinpyo Hong, Man Hyung Lee, Ho-Hwan Chun. Observability of Error States in GPS/INS Integration [C]. IEEE 0018-9545. 2005

[2] LIU Zhun. Key Technique Research on Modern Navigation System [D]. Postdoctoral Report, Beijing University of Aeronautics and Astronautics, 2003

[3] WANG Zheng-ming, YI Dong-yun, ZHOU Hai-yin, et. Calibration and Evaluation of Trajectory Tracking Data [M]. Publishing Company of Nation University of Defense Technology, 1999

[4] WEN Yuan-Lan. Research on Satellite Precision Orbit Robust Estimation [J]. Chinese Journal of Space Science, 2001, 21(4): 341350 (in Chinese) 
[5] FAN Shi-wei, YI Dong-yun. The Uncertainties of System Error and Conversion of Parameters Estimation Precision [J]. Journal of Ballistics, 2006, 18(1): 30-34 (in Chinese)

[6] DENG Shi-jun. Measure Data Modeling and Semi-parameter Estimate [D]. Wuhan University, 2005
[7] Sun H Y, Wu Y. Semi-parametric regression and model refining [J]. Geospatial Information Science, 2002, 4(5): 10-13

[8] ZHOU Hai-yin. Researches on Theories and Models of Spatial Targets Tracking Data Fusion with Applications [D]. Nation University of Defense Technology, 2004 